\title{
Prevalence, Incidence and Clinical Outcomes of Epicardial Coronary Artery Disease Among Transthyretin Amyloidosis Cardiomyopathy Patients
}

\section{Rana Hassan}

University of Calgary Cumming School of Medicine

Robert J. H. Miller

Libin Cardiovascular Institute of Alberta

Jonathan G. Howlett

Libin Cardiovascular Institute of Alberta

James A. White

Libin Cardiovascular Institute of Alberta

Nowell M. Fine ( $\nabla$ nowellfine@gmail.com )

Libin Cardiovascular Institute of Alberta https://orcid.org/0000-0002-6546-6587

\section{Research Article}

Keywords: transthyretin amyloidosis cardiomyopathy, coronary artery disease, prevalence, mortality

Posted Date: February 21st, 2022

DOI: https://doi.org/10.21203/rs.3.rs-1340343/v1

License: (9) This work is licensed under a Creative Commons Attribution 4.0 International License. Read Full License 


\section{Abstract}

Background: Transthyretin amyloidosis cardiomyopathy (ATTR-CM) patients are often older and may be at risk for obstructive epicardial coronary artery disease (oeCAD). While ATTR-CM may cause small vessel coronary disease, the prevalence and clinical significance of oeCAD is not well described.

Methods and Results: The prevalence and incidence of oeCAD and its association with all-cause mortality and hospitalization among 133 ATTR-CM patients with $\geq 1$-year follow-up was evaluated. The mean age was $78 \pm 9$ years, $119(89 \%)$ were male, $116(87 \%)$ had wild-type and $17(13 \%)$ had hereditary subtypes. Seventy-two (54\%) patients underwent oeCAD investigations, with $30(23 \%)$ receiving a positive diagnosis. Among these patients, 23 (77\%) were diagnosed prior to ATTR-CM diagnosis, 6 (20\%) at the time of ATTR-CM diagnosis, and 1 (3\%) after ATTR-CM diagnosis. Baseline characteristics between patients with and without oeCAD were similar. Among patients with oeCAD, only $2(7 \%)$ required additional investigations, intervention or hospitalization after ATTR-CM diagnosis. After a median followup of 27 months there were $37(28 \%)$ deaths occurring in 5 patients with oeCAD $(17 \%)$ and 32 patients without oeCAD (31\%). Fifty-six (42\%) patients required hospitalization, including 10 patients with oeCAD (33\%) and 46 patients without oeCAD (45\%). There was no significant difference in the rates of death or hospitalization among ATTR-CM patients with and without oeCAD, and oeCAD was not significantly associated with either outcome by regression analysis.

Conclusions: While oeCAD is prevalent in ATTR-CM patients, this diagnosis is frequently known at time of ATTR-CM diagnosis and is not associated with subsequent death or hospitalization.

\section{Introduction}

Transthyretin amyloidosis cardiomyopathy (ATTR-CM) is an important and underrecognized cause of heart failure (HF). ${ }^{1}$ Both ATTR-CM subtypes, the hereditary type (hATTR) caused by a transthyretin (TTR) gene mutation, and especially the wild-type (wtATTR), an age-related disorder occurring in the absence of a TTR gene mutation, often occur in older patients. ${ }^{2}$ As a result, many ATTR-CM patients have a significant burden of age-related comorbid conditions, including cardiovascular comorbidities. Patients with ATTR-CM can develop biventricular heart failure (HF) that progresses to a restrictive cardiomyopathy phenotype with variable systolic function. ${ }^{1}$ Other recognized cardiovascular manifestations may include atrial arrhythmias, conduction system disease, aortic valve stenosis, and autonomic dysfunction. ${ }^{1}$

ATTR-CM patients may present with symptoms of ischemic heart disease. This has been frequently attributed to intramural microvascular amyloid fibril deposition leading to microvascular ischemia. ${ }^{3-5}$ While histopathologic studies have identified the presence of amyloid deposits in epicardial coronary artery walls, this does not commonly lead to obstructive disease resulting in a clinical diagnosis of coronary artery disease. ${ }^{4,6}$ Given their frequently older age, ATTR-CM patients may have a meaningful prevalence of concurrent atherosclerotic obstructive epicardial coronary artery disease (oeCAD). Furthermore, it has been speculated that epicardial coronary artery amyloid deposits may accelerate 
existing atherosclerotic epicardial coronary disease. ${ }^{7,8}$ The prevalence and incidence of a clinical diagnosis of oeCAD in patients with ATTR-CM, along with its prognostic significance, is not well described. This may have direct relevance for the management of ATTR-CM patients because some medications frequently used for the treatment of oeCAD, such as beta-blockers and afterload reducing agents, are often poorly tolerated. ${ }^{1}$ Approval of the TTR-stabilizing agent tafamidis may improve the prognosis for ATTR-CM patients, increasing the relevance of concurrent oeCAD in this population. ${ }^{9}$ The purpose of this analysis was to describe the prevalence of clinically diagnosed oeCAD among ATTR-CM patients and its respective association with adverse clinical outcomes.

\section{Methods}

\section{Study Population}

Consecutive patients followed at the Cardiac Amyloidosis Clinic at our institution with a diagnosis of ATTR-CM from November 2016 to January 2021 were included in this retrospective cohort analysis. Patients with both ATTR subtypes were included if they met the following criteria; 1) exclusion of lightchain ( $\mathrm{AL}$ ) amyloidosis through the absence of serum and urine monoclonal protein, 2) evidence of cardiac amyloidosis by either myocardial biopsy or positive technetium-99m-pyrophosphate nuclear scintigraphy defined by grade 2-3 myocardial uptake or heart-to-contralateral lung ratio $>1.5$, as previously described, ${ }^{10}$ and 3 ) either hATTR or wtATTR based upon results of genetic testing or proteomic analysis by mass spectrometry performed on biopsy tissue samples. Patients with non-ATTR subtypes of amyloidosis or those with $<12$ months clinical follow-up were excluded. Clinical, medication, biochemical and cardiac imaging data were collected at the time of ATTR-CM diagnosis. This study was approved by the University of Calgary Research Ethics Board, and the requirement for informed written patient consent was waived.

\section{Coronary Artery Disease}

All patients included underwent review of medical records for evidence of oeCAD by two study investigators, including symptom history, healthcare encounters such as ambulatory clinic visits, Emergency Department visits and hospitalizations, and cardiac investigation findings such as electrocardiogram (ECG), cardiac biomarker (troponin and N-terminal pro-B-type natriuretic peptide, NTproBNP), ECG stress test, stress imaging, coronary computed tomography angiography (CCTA), invasive coronary angiography, history of acute coronary syndrome (ACS) or myocardial infarction (MI), and/or coronary artery revascularization. The clinical indication for oeCAD evaluation, in addition to the temporal relation with ATTR-CM diagnosis (occurring before, after, or simultaneous with) was also collected.

As patients with ATTR-CM often have clinical characteristics and/or non-invasive investigation result findings that resemble oeCAD (such as chest pain, chronically elevated troponin levels, and anterior Qwaves on ECG), a strict definition of oeCAD was used for this analysis. A diagnosis of CAD required $\geq 1$ 
of the following criteria: 1 ) prior history of coronary artery revascularization by either percutaneous coronary intervention (PCI) and/or coronary artery bypass grafting (CABG), 2) obstructive epicardial coronary artery stenosis of $\geq 70 \%$ by CCTA or invasive coronary angiography, or $\geq 50 \%$ of the left main coronary artery. ${ }^{11}$ Among patients with a prior history of ACS/MI, all had subsequent confirmatory invasive coronary angiography.

\section{Statistical Analysis}

Categorical variables are reported as frequency (percentage) and compared with a Chi-square or Fisher exact test. Continuous variables are reported as mean (standard deviation, SD) and compared with a student's t-test if normally distributed or as the median (interquartile range, IQR) and compared with a Wilcoxon rank sum test. Correlations between the presence of oeCAD and clinical, ATTR-related, medication, biochemical and imaging characteristics were examined using logistic regression analysis. The primary outcome was all-cause mortality, and the secondary outcome was all-cause hospitalization, further categorized as cardiovascular and non-cardiovascular hospitalization. Kaplan-Meier survival curves were constructed, and the log-rank test was used for comparison between ATTR-CM patients with and without oeCAD. For time to event analyses, event time was calculated as time from ATTR-CM diagnosis to study outcome. Univariable Cox proportional hazards regression modeling was used to identify whether the presence of oeCAD was associated with study outcomes all-cause death and a composite of all-cause death or all-cause hospitalization, with hazard ratios (HR) and 95\% confidence intervals (Cls) reported. All probability values were 2-sided, and a value of $\leq 0.05$ was considered statistically significant. Statistical analyses were performed using Stata software version 17.0 (College Station, Texas).

\section{Results}

\section{Study Population}

Out of 162 eligible ATTR-CM patients, 11 were excluded due to insufficient available data in their medical records to exclude or confirm a diagnosis of oeCAD, and 18 were excluded for $<12$ months follow-up, for a final study population of 133. Baseline characteristics of the study population and for those with wtATTR versus hATTR subtypes are shown in Table 1. The majority of ATTR-CM patients were male and had wtATTR. Among hATTR patients, the most common TTR gene mutations were pV142I, pT80A and pV30M. Other mutations represented in the cohort include one patient each with a pl127V, pl104T and pl88L mutation. wtATTR patients were significantly older, had a higher NYHA functional class and a higher proportion taking diuretics, while a significantly higher proportion of hATTR patients were taking an ATTR disease modifying agent (Table 1).

Table 1. Baseline characteristics of the study population and for patients with wtATTR and hATTR. 


\begin{tabular}{|c|c|c|c|c|}
\hline Parameter & $\begin{array}{l}\text { Total } \\
(\mathrm{N}=133)\end{array}$ & $\begin{array}{l}\text { wtATTR } \\
(n=116)\end{array}$ & $\begin{array}{l}\text { hATTR } \\
(n=17)\end{array}$ & p-value \\
\hline \multicolumn{5}{|l|}{ Clinical Characteristics } \\
\hline Age (years) & $78 \pm 9$ & $80 \pm 8$ & $66 \pm 8$ & $<0.01$ \\
\hline Male & $119(89 \%)$ & $106(91 \%)$ & $13(76 \%)$ & 0.08 \\
\hline Body mass index $\left(\mathrm{kg} / \mathrm{m}^{2}\right)$ & $27 \pm 4$ & $26 \pm 5$ & $27 \pm 5$ & 0.94 \\
\hline NYHA functional class III-IV & $72(54 \%)$ & $67(58 \%)$ & $5(29 \%)$ & 0.04 \\
\hline Atrial fibrillation & $48(36 \%)$ & $44(38 \%)$ & $4(24 \%)$ & 0.29 \\
\hline \multicolumn{5}{|l|}{ hATTR mutation } \\
\hline pV142I & $5(4 \%)$ & - & $5(29 \%)$ & - \\
\hline pV50M & $5(4 \%)$ & - & $5(29 \%)$ & - \\
\hline pT80A & $4(3 \%)$ & - & $4(24 \%)$ & - \\
\hline other & $3(2 \%)$ & - & $3(18 \%)$ & - \\
\hline \multicolumn{5}{|l|}{ Medications } \\
\hline Diuretics & $90(68 \%)$ & $84(72 \%)$ & $6(35 \%)$ & $<0.01$ \\
\hline Anticoagulation & $53(40 \%)$ & $49(42 \%)$ & $4(24 \%)$ & 0.19 \\
\hline ATTR therapy & $78(59 \%)$ & $64(55 \%)$ & $14(82 \%)$ & 0.04 \\
\hline Tafamidis & $71(53 \%)$ & $64(55 \%)$ & $7(41 \%)$ & - \\
\hline Inotersen & $2(2 \%)$ & 0 & $2(12 \%)$ & - \\
\hline Patisiran & $5(4 \%)$ & 0 & $5(29 \%)$ & - \\
\hline \multicolumn{5}{|l|}{ Biochemical } \\
\hline Troponin-T (ng/L) & $61(39,87)$ & $63(49,92)$ & $50(29,63)$ & 0.07 \\
\hline NTproBNP (ng/L) & $1984(871,2537)$ & $2171(1082,2579)$ & $1688(767,1936)$ & 0.20 \\
\hline eGFR (mL/min/1.73m²) & $49(43,52)$ & $47(42,54)$ & $49(44,56)$ & 0.83 \\
\hline \multicolumn{5}{|l|}{ Echocardiographic } \\
\hline LV mass index $\left(\mathrm{g} / \mathrm{m}^{2}\right)$ & $156(139,170)$ & $159(145,191)$ & $147(133,152)$ & 0.13 \\
\hline LV ejection fraction (\%) & $53(48,56)$ & $51(46,57)$ & $56(50,61)$ & 0.38 \\
\hline LV GLS (\%) & $13.6(11.8,16.7)$ & $13.1(11.5,16.0)$ & $14.2(11.8,17.2)$ & 0.53 \\
\hline
\end{tabular}


mean ( \pm standard deviation) or median (interquartile range). ATTR-CM-transthyretin amyloidosis cardiomyopathy, eGFR-estimated glomerular filtration rate, GLS-global longitudinal systolic strain, hATTR-hereditary transthyretin amyloidosis, LV-left ventricle, NTproBNP-N-terminal pro-B-type natriuretic peptide, NYHA-New York Heart Association, wtATTR-wild-type transthyretin amyloidosis.

\section{Evaluation, Prevalence and Incidence of oeCAD}

Overall, there were 72 (54\%) ATTR-CM patients who underwent some form of invasive or non-invasive investigation for the assessment of known or suspected oeCAD. The indications for oeCAD evaluation are listed in Table 2. Dyspnea and chest pain were the most common indications, followed by patients presenting with ACS and those being investigated for abnormal resting ECG. Among these 72 ATTR-CM patients, 37 (51\%) underwent investigations prior to being diagnosed with ATTR-CM (range 21 months to 38 years prior to ATTR-CM diagnosis), of which 23 (62\%) were confirmed to have oeCAD. There were 33 ( $46 \%$ of those ever investigated for oeCAD) ATTR-CM patients who underwent investigations as part of their ATTR-CM diagnostic work-up, of which only $6(18 \%)$ were confirmed to have oeCAD. Only 2 (3\% of those ever investigated for oeCAD) patients underwent oeCAD investigation following their ATTR-CM diagnosis, including one patient who had syncope and a negative oeCAD work-up, and another presenting with ACS and found to have multi-vessel oeCAD who was subsequently managed medically (further details provided below). Figure 1 presents a flow chart for the study cohort, describing the proportion of patients who underwent an evaluation for oeCAD at varying time points during their ATTR-CM care pathway, and whether the investigations placed them in a positive or negative category for oeCAD. 
Table 2

Comparison of baseline characteristics for ATTR-CM patients with and without oeCAD.

\begin{tabular}{|c|c|c|c|}
\hline Parameter & $\begin{array}{l}\text { oeCAD } \\
(n=30)\end{array}$ & $\begin{array}{l}\text { No oeCAD } \\
(n=103)\end{array}$ & p-value \\
\hline \multicolumn{4}{|l|}{ Clinical Characteristics } \\
\hline Age (years) & $79 \pm 7$ & $78 \pm 10$ & 0.74 \\
\hline Male & $28(93 \%)$ & $91(88 \%)$ & 0.68 \\
\hline Body mass index $\left(\mathrm{kg} / \mathrm{m}^{2}\right)$ & $29 \pm 5$ & $27 \pm 4$ & 0.81 \\
\hline NYHA functional class III-IV & $20(67 \%)$ & $52(50 \%)$ & 0.14 \\
\hline Atrial fibrillation & $14(47 \%)$ & $34(33 \%)$ & 0.2 \\
\hline \multicolumn{4}{|l|}{ ATTR characteristics } \\
\hline wtATTR & $28(93 \%)$ & $88(85 \%)$ & 0.36 \\
\hline hATTR & $2(7 \%)$ & $15(15 \%)$ & \\
\hline pV142I & $1(3 \%)$ & $4(4 \%)$ & 0.99 \\
\hline pV50M & 0 & $5(5 \%)$ & 0.59 \\
\hline pT80A & $1(3 \%)$ & $3(3 \%)$ & 0.99 \\
\hline other & 0 & $3(3 \%)$ & 0.99 \\
\hline \multicolumn{4}{|l|}{ Medications } \\
\hline Diuretics & $22(73 \%)$ & $68(66 \%)$ & 0.51 \\
\hline Anticoagulation & $16(53 \%)$ & $37(36 \%)$ & 0.09 \\
\hline ATTR therapy & $21(70 \%)$ & $57(55 \%)$ & 0.21 \\
\hline Tafamidis & $21(70 \%)$ & $50(48 \%)$ & 0.06 \\
\hline Inotersen & 0 & $2(2 \%)$ & 0.99 \\
\hline Patisiran & 0 & $5(5 \%)$ & 0.59 \\
\hline \multicolumn{4}{|l|}{ Biochemical } \\
\hline Troponin-T (ng/L) & $71(46,85)$ & $56(31,63)$ & 0.22 \\
\hline NTproBNP (ng/L) & $2218(1672,2625)$ & $1794(738,2041)$ & 0.17 \\
\hline $\operatorname{eGFR}\left(\mathrm{mL} / \mathrm{min} / 1.73 \mathrm{~m}^{2}\right)$ & $47(41,50)$ & $50(44,52)$ & 0.91 \\
\hline
\end{tabular}




\begin{tabular}{|lllc|}
\hline Parameter & $\begin{array}{l}\text { oeCAD } \\
(\mathbf{n = 3 0 )}\end{array}$ & $\begin{array}{l}\text { No oeCAD } \\
(\mathbf{n = 1 0 3 )}\end{array}$ & p-value \\
\hline LV mass index $\left(\mathrm{g} / \mathrm{m}^{2}\right)$ & $167(144,189)$ & $149(131,162)$ & 0.23 \\
\hline LV ejection fraction $(\%)$ & $48(45,54)$ & $55(49,58)$ & 0.19 \\
\hline LV GLS $(\%)$ & $12.9(10.8,14.6)$ & $14.3(12.5,17.2)$ & 0.66 \\
\hline $\begin{array}{l}\text { Categorical variables are reported as frequency (percentage) } \\
\text { mean }( \pm \text { standard deviation) or median (interquartile range). ATTR-CM-transthyretin amyloidosis } \\
\text { cardiomyopathy, eGFR-estimated glomerular filtration rate, GLS-global longitudinal systolic strain, }\end{array}$ \\
$\begin{array}{l}\text { hATTR-hereditary transthyretin amyloidosis, LV-left ventricle, NTproBNP-N-terminal pro-B-type } \\
\text { natriuretic peptide, NYHA-New York Heart Association, oeCAD-obstructive epicardial coronary artery } \\
\text { disease, wtATTR-wild-type transthyretin amyloidosis. }\end{array}$ \\
\hline
\end{tabular}

Table 3

Indications for oeCAD evaluation among ATTR-CM patients.

\begin{tabular}{|ll|}
\hline Parameter & Total $(\mathbf{N}=\mathbf{7 2})$ \\
\hline Dyspnea & $20(28 \%)$ \\
\hline Chest pain & $19(26 \%)$ \\
\hline Acute coronary syndrome & $18(25 \%)$ \\
\hline NSTEMI & $12(67 \%)$ \\
\hline STEMI & $6(33 \%)$ \\
\hline Abnormal ECG & $6(8 \%)$ \\
\hline Pre-cardiac surgery / procedure & $4(6 \%)$ \\
\hline Syncope & $3(4 \%)$ \\
\hline Ventricular arrhythmia & $2(3 \%)$ \\
\hline $\begin{array}{l}\text { ATTR-CM-transthyretin amyloidosis cardiomyopathy, ECG-electrocardiogram, NSTEMI-non-ST- } \\
\text { segment elevation myocardial infarction, oeCAD-obstructive coronary artery disease, STEMI-ST- } \\
\text { segment elevation myocardial infarction. }\end{array}$ \\
\hline
\end{tabular}

There were 48 ATTR-CM patients (36\% of the total study population) whose oeCAD evaluation included invasive coronary angiography, among whom $30(65 \%)$ received a confirmed diagnosis of oeCAD (including 18 patients presenting with ACS), and 18 patients (35\%) identified to have no oeCAD. A total of twenty-three (17\% of the total study population) patients had myocardial perfusion imaging performed, including 22 (96\%) who had single photon emission computed tomography and 1 (4\%) stress-perfusion cardiac magnetic resonance imaging. None of these patients demonstrated more than a mild/small perfusion defect. Three (13\%) of these 23 patients went on to invasive testing and had oeCAD confirmed by angiography in the setting of persistent symptoms, and the remainder had no further investigations for 
oeCAD. Twelve (52\%) of the patients who underwent myocardial perfusion imaging had it performed as part of the work-up for their clinical presentation with ATTR-CM, all demonstrating either normal perfusion or a mild/small perfusion defect.

\section{Characteristics ATTR-CM Patients with oeCAD}

Figure 2 reports the prevalence of oeCAD among ATTR-CM patients. There were 30 (23\% of the total study population, $42 \%$ of those evaluated for oeCAD) ATTR-CM patients with confirmed oeCAD (all confirmed by invasive coronary angiography). Baseline characteristics for ATTR-CM patients with and without oeCAD are shown in Table 2. All clinical, ATTR specific, medication, biochemical and imaging characteristics were statistically similar between groups. Figure 3 compares the prevalence of oeCAD among select ATTR-CM patient subgroups.

Among patients with confirmed oeCAD, $23(77 \%)$ received this diagnosis before being diagnosed with ATTR-CM, including 17 with prior ACS (11 [65\%] with NSTEMI and 6 [35\%] with STEMI) and 18 with prior revascularization (11 [61\%] with $\mathrm{PCl}$ and 7 [39\%] with CABG). Of note, among patients with a prior diagnosis of oeCAD, none required further oeCAD-related investigations, interventions or hospitalization after being diagnosed with ATTR-CM. There were 6 ( $20 \%$ of patients with confirmed oeCAD) patients diagnosed with oeCAD during their work-up for ATTR-CM. One patient went on to have CABG and the other 5 patients were managed medically. Again, none of those 6 patients required further investigations, interventions or hospitalization for oeCAD in follow-up. One ATTR-CM patient was diagnosed with oeCAD 13-months after their diagnosis with ATTR-CM, presenting to hospital with NSTEMI and invasive coronary angiography confirming multi-vessel oeCAD. This patient was managed medically and has not required subsequent investigations, interventions or hospitalizations for oeCAD. Among all clinical, ATTR-specific, medication, biochemical and imaging variables listed in Table 1, none were significantly associated with a diagnosis of oeCAD among ATTR-CM patients.

\section{Outcomes of ATTR-CM Patients With and Without oeCAD}

At a median follow-up of 27 months (interquartile range 18,35 months) 37 ( $28 \%$ of the total study population) ATTR-CM patients died, including $5(17 \%)$ deaths of patients with oeCAD and $32(31 \%)$ deaths of ATTR-CM patients without oeCAD. Figure 4 demonstrates Kaplan-Meier survival curves for ATTR-CM patients with and without oeCAD, demonstrating no significant difference in survival between groups (log-rank $p=0.54)$. Using Cox proportional hazards modeling, the presence of oeCAD was not significantly associated with mortality $(\mathrm{HR} 0.74,95 \% \mathrm{Cl} 0.29-1.92, \mathrm{p}=0.54)$.

There were 56 ( $42 \%$ of the total study population) ATTR-CM patients hospitalized during the follow-up period, including 10 (18\%) patients with oeCAD (6 [60\%] cardiovascular hospitalizations and 4 [40\%] noncardiovascular hospitalizations), and 46 (82\%) patients without oeCAD (29 [63\%] cardiovascular hospitalizations and 17 [37\%] non-cardiovascular hospitalizations). Figure 4 demonstrates Kaplan-Meier curves for hospitalization of ATTR-CM patients with and without oeCAD, demonstrating no significant difference in survival between groups (log-rank $p=0.65)$. Using Cox proportional hazards modeling, the 
presence of oeCAD was not significantly associated with the composite outcome of death or hospitalization (HR 0.86, 95\% Cl 0.42-1.73, $\mathrm{p}=0.65$ ).

\section{Discussion}

This study is the first to describe the prevalence of oeCAD among patients with ATTR-CM and its associations to major clinical outcomes. The main findings are as follows: 1) approximately one quarter of ATTR-CM patients in our cohort had concurrent oeCAD, the majority of whom were diagnosed prior to ATTR-CM diagnosis, 2) upon clinical presentation with ATTR-CM, most patients underwent some form of oeCAD-related investigation, the majority of these being negative, 3 ) among ATTR-CM patients with oeCAD, the majority did not exhibit manifestations of this at the time of ATTR-CM diagnosis and during follow-up, and 4) a diagnosis of oeCAD was not associated with adverse outcomes in ATTR-CM patients. Overall, these findings suggest that, while oeCAD is prevalent among patients with ATTR-CM, it typically precedes ATTR-CM diagnosis and does significantly impact prognosis for most patients.

Cardiac amyloidosis is recognized to be associated with intramural microvascular disease in the absence of oeCAD, and much of this evidence comes from histopathologic studies of AL amyloidosis patients. ${ }^{3}$ This small vessel disease can result in symptoms of angina, and may contribute to unstable coronary syndromes or progressive HF. ${ }^{7}$ Multiple reports have described cardiac amyloidosis presenting with signs and/or symptoms of ischemic heart disease, ${ }^{12,13}$ It has been reported that between $10-20 \%$ of cardiac amyloidosis patients initially present with symptoms of chest pain, and that up to two-thirds of patients have significant intramural coronary amyloid deposits, many associated with focal microscopic changes of ischemic injury. ${ }^{14,15}$ Older pathologic studies describe the prevalence of intramural coronary artery amyloid deposits as much lower in ATTR-CM compared with AL cardiac amyloidosis, ${ }^{16-18}$ while more recent studies have confirmed coronary amyloid deposits in ATTR-CM patients. ${ }^{6}$ Perivascular and interstitial myocardial deposits may cause compression of coronary microvasculature that can also contribute to symptoms of ischemic heart disease. ${ }^{19}$ Significant coronary microvascular dysfunction was demonstrated by Dorbala, et al in a mixed cardiac amyloidosis subtype (ATTR and AL) cohort of patients without epicardial coronary artery disease undergoing $\mathrm{N}-13$ ammonia positron emission tomography, and patients had reduced peak vasodilator stress myocardial blood flow and flow reserve. ${ }^{19}$

While amyloid infiltration of the epicardial coronary artery wall is also common in histopathologic studies, it generally does not result in obstructive disease. ${ }^{14}$ Our study is consistent with earlier reports that a clinical diagnosis of oeCAD is rare once patients receive a diagnosis of ATTR-CM,, 46 and that most patients with oeCAD are diagnosed prior to receiving a ATTR-CM diagnosis. Because of similarities in clinical presentation between oeCAD and cardiac amyloidosis, many ATTR-CM patients undergo investigation for oeCAD at the time of presentation. Beyond similar signs and symptoms, many cardiac amyloidosis patients have ECG abnormalities that may mimic oeCAD, and many have chronically elevated troponin values. In our study, the majority of investigations for oeCAD at the time of ATTR-CM diagnosis were negative. It has been postulated that cardiac amyloidosis may accelerate pre-existing 
atherosclerotic epicardial coronary artery diease via the deposition of amyloid deposits around coronary vessels, ${ }^{7,8}$ however the findings from our study do not support this in patients with ATTR-CM. Mortality and hospitalization rate were similar between patients and without oeCAD, and very few patients required further investigations for oeCAD after ATTR-CM diagnosis. Although beyond the scope of this study to confirm, these findings might suggest that medications commonly used to treat oeCAD that are poorly tolerated in patients with ATTR-CM, especially beta-blockers, may be safely reduced or even discontinued in patients with a prior oeCAD diagnosis, although this requires further research, and a cautious approach to titration of these medications in this setting is still recommended.

\section{Limitations}

There are a number of important limitations to our analysis. This was a single-center, retrospective, observational cohort study, and therefore the presence of bias cannot be excluded. Patients did not undergo evaluation for microvascular dysfunction, and therefore the prevalence of this and its impact on our results is uncertain. Similarly, autopsy data was not available for deceased patients, and so correlation of clinical with histopathologic findings cannot be performed, although several previous studies have examined this. Lastly, our report lacks detailed descriptions of medical management changes for ATTR-CM patients, including anti-anginal agents often used to treat oeCAD. A recent report describing rates of beta-blocker prescription among a mixed subtype cohort of cardiac amyloidosis patients found that their use was not infrequent, with approximately $1 / 3$ of patients continuing to take them after diagnosis, the majority for treatment of arrhythmia. ${ }^{20}$

\section{Conclusions}

oeCAD is prevalent among ATTR-CM patients, however is typically known at time of ATTR-CM diagnosis. Characteristics of ATTR-CM patients with and without oeCAD are similar, and both appear to have similar prognosis with respect to both mortality and hospitalization. ATTR-CM patients with oeCAD typically did not require additional investigations or oeCAD-related interventions during follow-up. While confirmation is needed by larger prospective studies, this report suggests that the presence of oeCAD does not significantly influence clinical outcomes in patients with ATTR-CM.

\section{Abbreviations}

ACS-acute coronary syndrome

ATTR-CM-transthyretin amyloidosis cardiomyopathy

CABG-coronary artery bypass grafting

ECG-electrocardiogram

hATTR-hereditary transthyretin amyloidosis 
MI-myocardial infarction

oeCAD-obstructive epicardial coronary artery disease

PCl-percutaneous coronary intervention

TTR-transthyretin protein

wtATTR-wild-type transthyretin amyloidosis

\section{Declarations}

Ethics approval and consent to participate: This study was approved by the University of Calgary Research Ethics Board (REB20-1319), and the requirement for informed written patient consent was waived.

Consent for publication: Not applicable.

Availability of data and materials: The datasets used and/or analyzed during the current study are available from the corresponding author on reasonable request.

Funding sources: None

Competing interests: Dr. Fine has received research support from Pfizer, Akcea/lonis, Eidos and Alnylam and consulting honoraria from Pfizer, Akcea/lonis and Alynylam. Dr. Miller has received research support from Pfizer and consulting honoraria from Pfizer and Alnylam. Dr White has received research support from Pfizer, Siemens Healthineers, and Circle Cardiovascular Inc., and holds shares in Cohesic Inc. The remaining authors have no relevant disclosures.

Author contributions: All authors have participated in the work and have reviewed and agree with the content of the article. R.H. and N.M.F. participated in study conception, design and data analysis and interpretation, and R.H., R.J.H.M., J.G.H, J.A.W. and N.M.F. participated in manuscript drafting, critical revision for intellectual content, and final approval.

Acknowledgements: We would like to acknowledge the nurses and staff of the Cardiac Amyloidosis Clinic at South Health Campus hospital (Alberta Health Services, Calgary Alberta Canada) for their tireless support and dedication to patient care.

\section{References}

1. Kittleson MM, Maurer MS, Ambardekar AV, et al. Cardiac Amyloidosis: Evolving Diagnosis and Management: A Scientific Statement From the American Heart Association. Circulation Jul. 
2020;142(1):e7-22. doi:10.1161/CIR.0000000000000792.

2. Maurer MS, Hanna M, Grogan M, et al. Genotype and Phenotype of Transthyretin Cardiac Amyloidosis: THAOS (Transthyretin Amyloid Outcome Survey). J Am Coll Cardiol. 2016;07(2):16172. doi:10.1016/j.jacc.2016.03.596. 68 ) .

3. Mueller PS, Edwards WD, Gertz MA. Symptomatic ischemic heart disease resulting from obstructive intramural coronary amyloidosis. Am J Med Aug. 2000;15(3):181-8. doi:10.1016/s00029343(00)00471-x. 109 ) .

4. Wittich CM, Neben-Wittich MA, Mueller PS, Gertz MA, Edwards WD. Deposition of amyloid proteins in the epicardial coronary arteries of 58 patients with primary systemic amyloidosis. Cardiovasc Pathol. 2007 Mar-Apr. 2007;16(2):75-8. doi:10.1016/j.carpath.2006.09.011.

5. Hashimura $\mathrm{H}$, Ishibashi-Ueda $\mathrm{H}$, Yonemoto $\mathrm{Y}$, et al. Late gadolinium enhancement in cardiac amyloidosis: attributable both to interstitial amyloid deposition and subendocardial fibrosis caused by ischemia. Heart Vessels Jun. 2016;31(6):990-5. doi:10.1007/s00380-015-0658-0.

6. Mohammed SF, Mirzoyev SA, Edwards WD, et al. Left ventricular amyloid deposition in patients with heart failure and preserved ejection fraction. JACC Heart Fail Apr. 2014;2(2):113-22. doi:10.1016/j.jchf.2013.11.004.

7. Singh V, Tiemeier A, Malhotra S. Coexistence of cardiac amyloidosis with coronary artery disease and the challenges in medical management. J Nucl Cardiol. 2019;06(3):790-6. doi:10.1007/s12350019-01644-9. 26 ) .

8. Beyene SS, Yacob O, Melaku GD, et al. Comparison of Patterns of Coronary Artery Disease in Patients With Heart Failure by Cardiac Amyloidosis Status. Cardiovasc Revasc Med Jun. 2021;27:31-5. doi:10.1016/j.carrev.2020.09.026.

9. Maurer MS, Schwartz JH, Gundapaneni B, et al. Tafamidis Treatment for Patients with Transthyretin Amyloid Cardiomyopathy. N Engl J Med. Sep 2018;379(11):1007-16. doi:10.1056/NEJMoa1805689.

10. Gillmore JD, Maurer MS, Falk RH, et al. Nonbiopsy Diagnosis of Cardiac Transthyretin Amyloidosis. Circulation. Jun 14 2016;133(24):2404-12. doi:10.1161/CIRCULATIONAHA.116.021612.

11. Rush CJ, Berry C, Oldroyd KG, et al. Prevalence of Coronary Artery Disease and Coronary Microvascular Dysfunction in Patients With Heart Failure With Preserved Ejection Fraction. JAMA Cardiol Jun 23 2021;doi:10.1001/jamacardio.2021.1825.

12. Nguyen HT, Nguyen CTH. Cardiac amyloidosis mimicking acute coronary syndrome: a case report and literature review. Eur Heart J Case Rep Dec. 2020;4(6):1-7. doi:10.1093/ehjcr/ytaa325.

13. Whitaker DC, Tungekar MF, Dussek JE. Angina with a normal coronary angiogram caused by amyloidosis. Heart Sep. 2004;90(9):e54. doi:10.1136/hrt.2004.038984.

14. Neben-Wittich MA, Wittich CM, Mueller PS, Larson DR, Gertz MA, Edwards WD. Obstructive intramural coronary amyloidosis and myocardial ischemia are common in primary amyloidosis. Am J Med. Nov 2005;118(11):1287. doi:10.1016/j.amjmed.2005.06.017.

15. Hongo $\mathrm{M}$, Yamamoto $\mathrm{H}$, Kohda $\mathrm{T}$, et al. Comparison of electrocardiographic findings in patients with AL (primary) amyloidosis and in familial amyloid polyneuropathy and anginal pain and their relation 
to histopathologic findings. Am J Cardiol Apr. 2000;01(7):849-53. doi:10.1016/s00029149(99)00879-6. 85 ) .

16. Smith TJ, Kyle RA, Lie JT. Clinical significance of histopathologic patterns of cardiac amyloidosis. Mayo Clin Proc. Aug 1984;59(8):547 - 55. doi:10.1016/s0025-6196(12)61493-1.

17. Olson LJ, Gertz MA, Edwards WD, et al. Senile cardiac amyloidosis with myocardial dysfunction. Diagnosis by endomyocardial biopsy and immunohistochemistry. N Engl J Med Sep. 1987;17(12):738-42. doi:10.1056/NEJM198709173171205. 317 ) .

18. Kyle RA, Spittell PC, Gertz MA, et al. The premortem recognition of systemic senile amyloidosis with cardiac involvement. Am J Med. Oct 1996;101(4):395-400. doi:10.1016/S0002-9343(96)00229-X.

19. Dorbala $S$, Vangala $D$, Bruyere $J$, et al. Coronary microvascular dysfunction is related to abnormalities in myocardial structure and function in cardiac amyloidosis. JACC Heart Fail Aug. 2014;2(4):358-67. doi:10.1016/j.jchf.2014.03.009.

20. Tini G, Cappelli F, Biagini E, et al. Current patterns of beta-blocker prescription in cardiac amyloidosis: an Italian nationwide survey. ESC Heart Fail Aug. 2021;8(4):3369-74. doi:10.1002/ehf2.13411.

\section{Figures}

Before ATTR-CM Dx

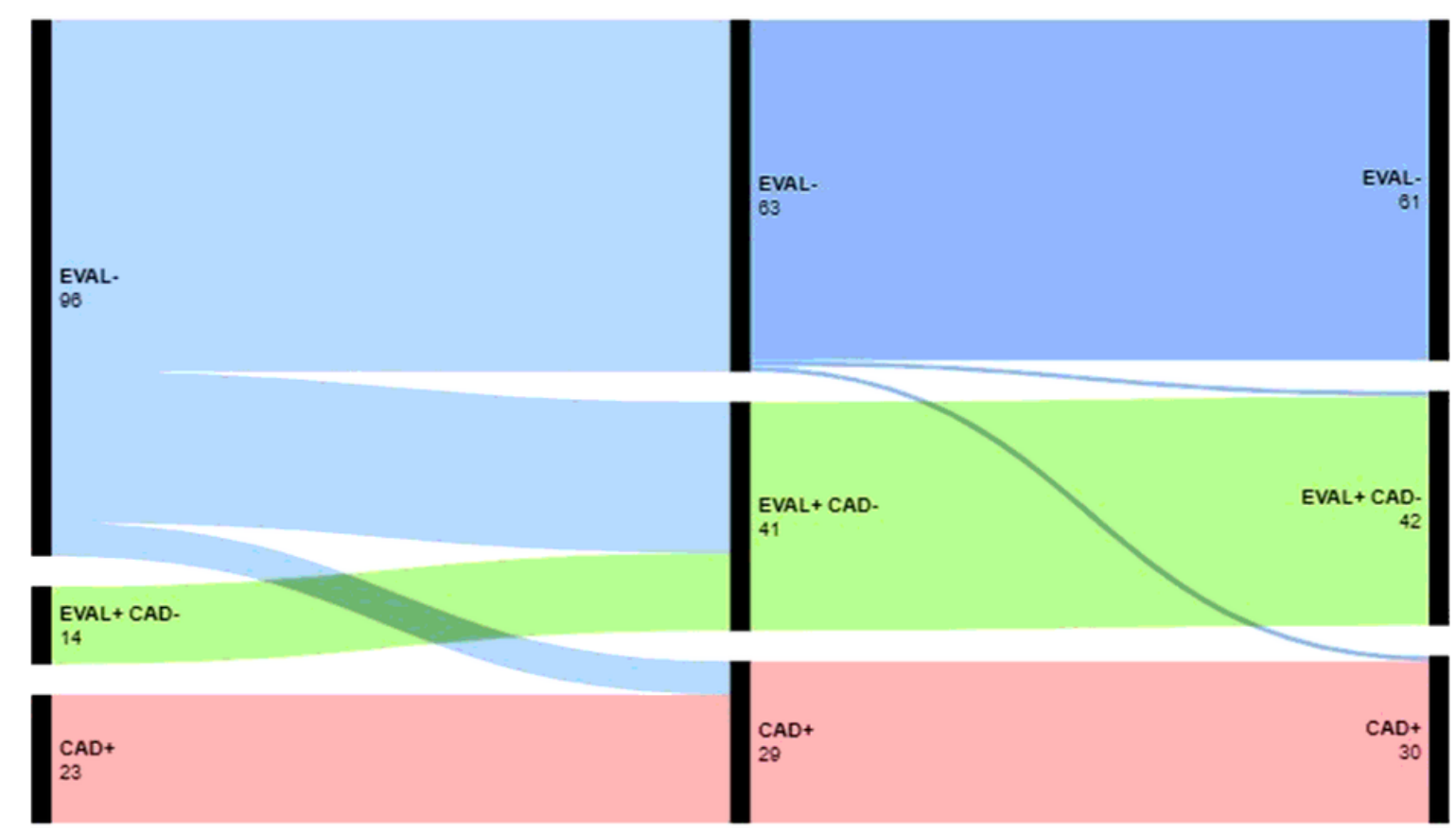

\section{Figure 1}

Study flow chart illustrating the time course of obstructive epicardial coronary artery disease (oeCAD) evaluation (EVAL) and diagnosis for transthyretin amyloidosis cardiomyopathy (ATTR-CM) patients, 
occurring either before, during or after ATTR-CM work-up/diagnosis (Dx). EVAL-indicates no oeCAD evaluation was performed, while EVAL+ indicates a oeCAD evaluation was performed. CAD- indicates that a diagnosis of oeCAD was excluded after evaluation, while CAD+ indicates a oeCAD diagnosis was confirmed (by invasive coronary angiography in all patients).

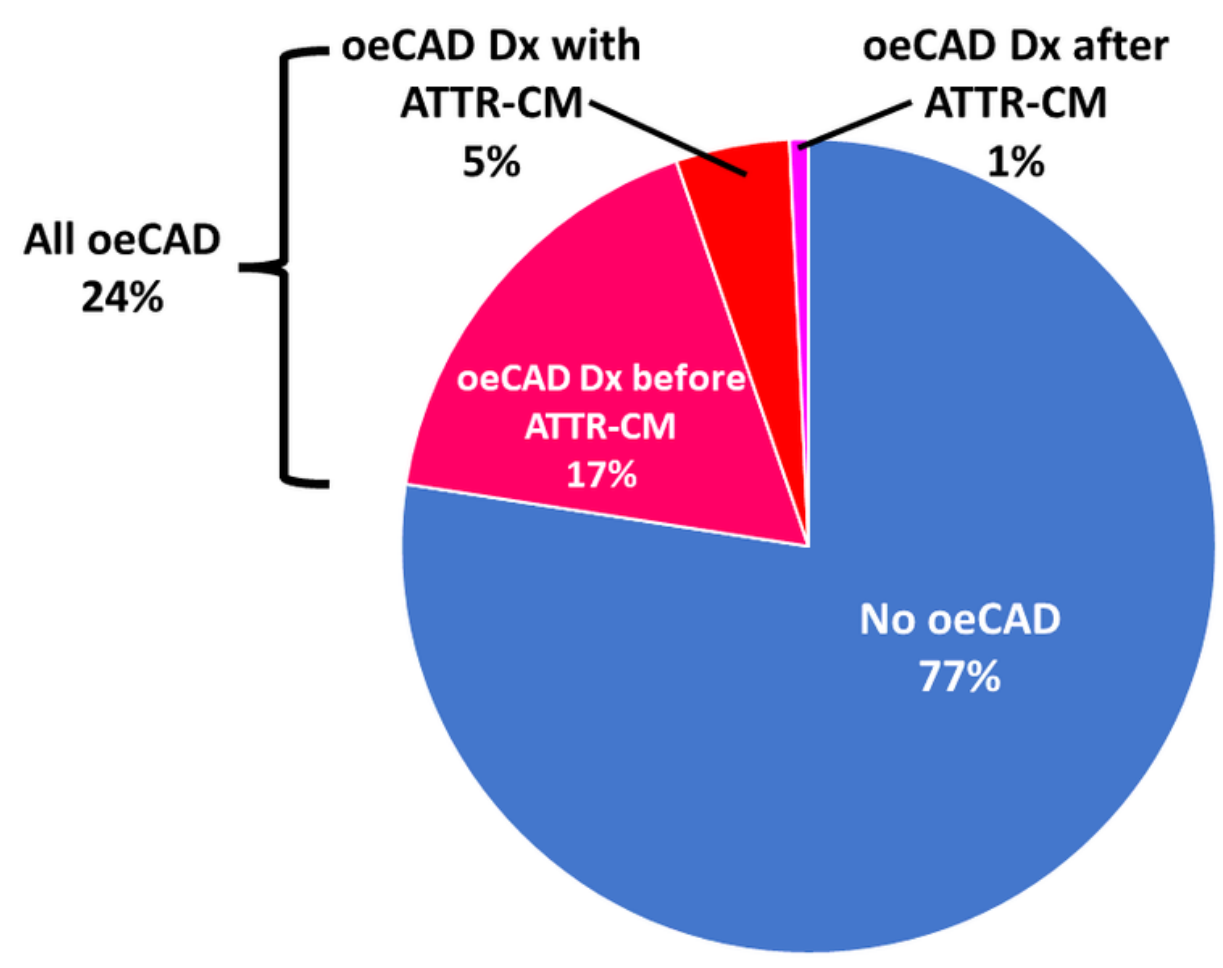

Figure 2

Prevalence of obstructive epicardial coronary artery disease (oeCAD) among transthyretin amyloidosis cardiomyopathy (ATTR-CM) patients, indicating the timing of oeCAD diagnosis as occurring either before, during or after their ATTR-CM work-up/diagnosis. 

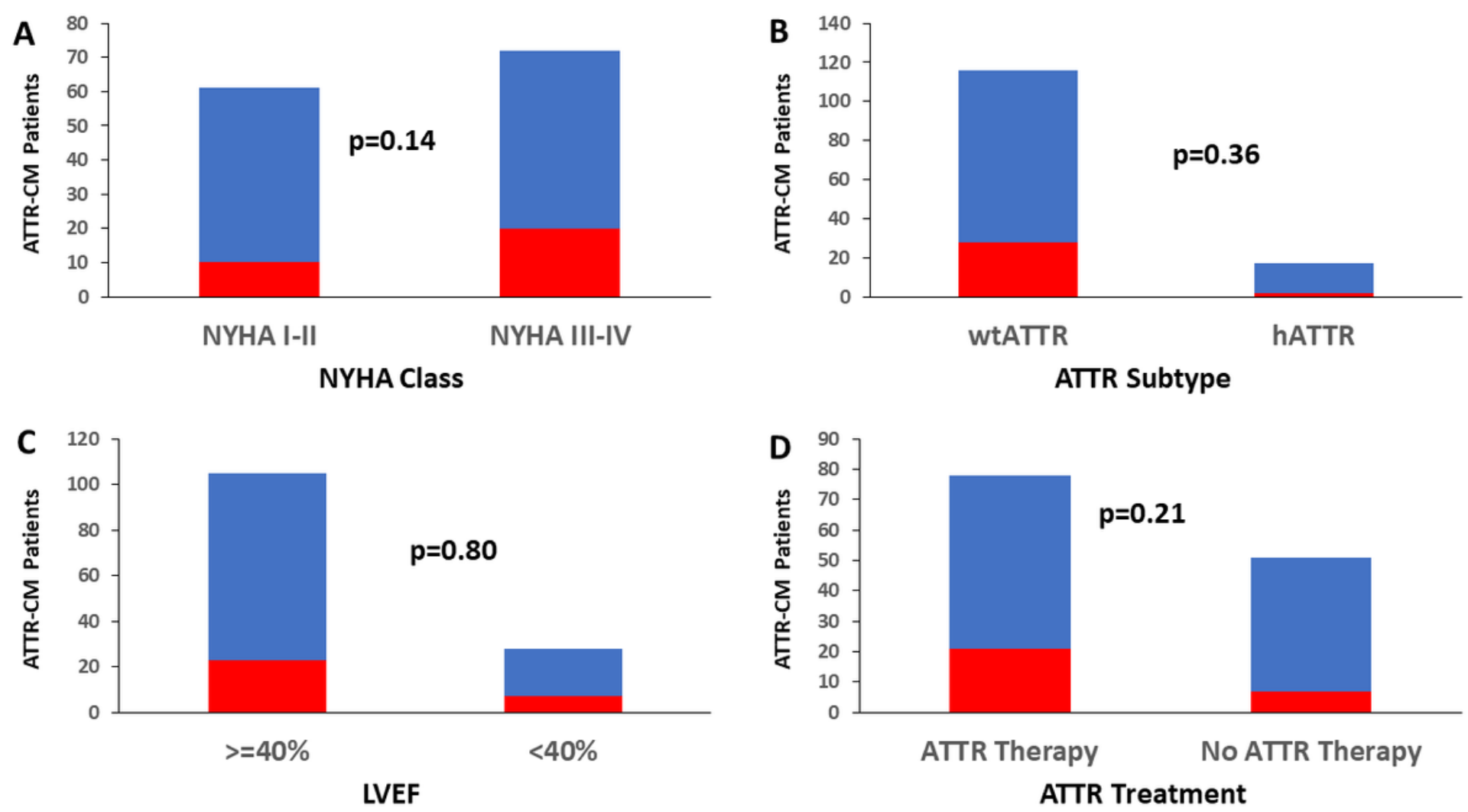

\section{Figure 3}

Comparison of the prevalence of obstructive epicardial coronary artery disease (oeCAD) among subgroups of transthyretin amyloidosis cardiomyopathy (ATTR-CM) patients. (A) New York Heart Association (NYHA) functional class, (B) ATTR subtype, wild-type (wtATTR) or hereditary (hATTR), (C) left ventricular ejection fraction (LVEF), and (D) use of disease modifying ATTR therapy. 


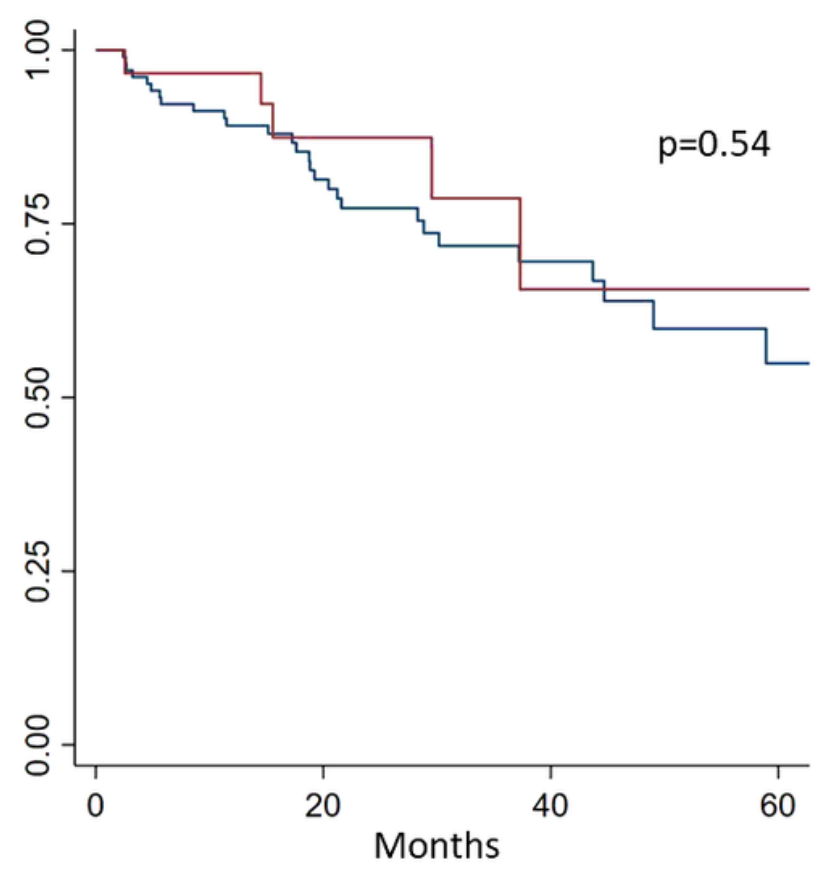

All-cause Mortality

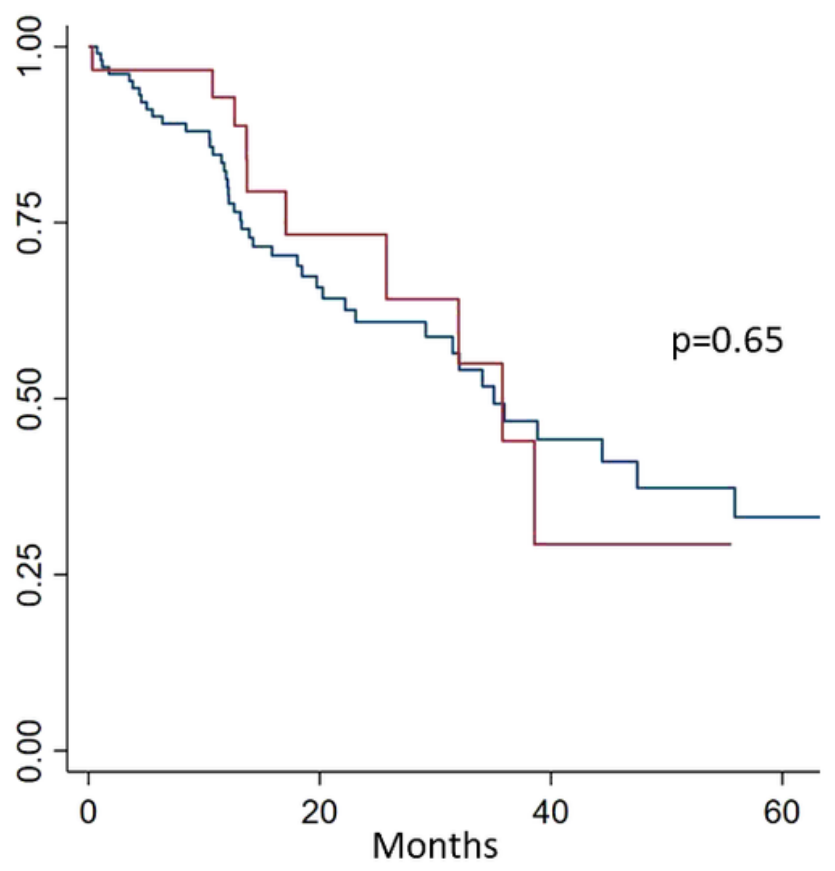

All-cause Hospitalizations

\section{Figure 4}

Kaplan-Meier curves (with corresponding log-rank test p-values) depicting the rates of all cause mortality (left) and all-cause hospitalizations (right) of transthyretin amyloidosis cardiomyopathy patients with (red) and without (blue) obstructive epicardial coronary artery disease. 